\title{
Finite-Amplitude Instability of the Compressible Laminar Wake. Strongly Amplified Disturbances
}

\author{
J. T. C. LID \\ Division of Engineering and Center for Fluid Dynamics, Brown University, \\ Providence, Rhode Island 02912 \\ AND \\ LESTER LEES \\ Firestone Flight Sciences Laboratory, California Institute of Technology, \\ Pasadena, California 91109
}

(Received 2 January 1970; final manuscript received 22 July 1970)

\begin{abstract}
The interaction between mean flow and finite-amplitude disturbances in certain experimentally observed unstable, compressible laminar wakes is considered theoretically without explicitly assuming small amplification rates. Boundary-layer form of the two-dimensional mean-flow momentum, kinetic energy and thermal energy equations and the time-averaged kinetic energy equation of spatially growing disturbances are recast into their respective von Kármán integral form which show the over-all physical coupling. The Reynolds shear stresses couple the mean flow and disturbance kinetic energies through the conversion mechanism familiar in low-speed flows. Both the mean flow and disturbance kinetic energies are coupled to the mean-flow thermal energy through their respective viscous dissipation. The work done by the disturbance pressure gradients gives rise to an additional coupling between the disturbance kinetic energy and the mean-flow thermal energy. The compressibility transformation suggested by work on turbulent shear flows is not applicable to this problem because of the accompanying ad hoc assumptions about the disturbance behavior. The disturbances of a discrete frequency which corresponds to the most unstable fundamental component, are first evaluated locally. Subsequent mean-flow and disturbance profile-shape assumptions are made in terms of a mean-flow-density Howarth variable. The compressibility transformation, which cannot convert this problem into a form identical to the low-speed problem of Ko, Kubota, and Lees because of the compressible disturbance quantities, nevertheless, yields a much simplified description of the mean flow.
\end{abstract}

\section{INTRODUCTION}

Recently, several wind-tunnel experiments on the details of the "transition" region of two-dimensional high-speed wake flows have appeared $d^{1-3}$ which have stimulated theoretical consideration of the finiteamplitude instability of the compressible laminar wake. ${ }^{4}$ In this earlier paper, ${ }^{4}$ one of the present authors extended the Stuart ${ }^{5}$ and Watson ${ }^{6,7}$ lowspeed, weakly nonlinear theory to a corresponding theory for a compressible fluid, with the explicit assumption of small amplification rates. This theory, within the limitations of the assumptions made, enables one to calculate the detailed distributions of the mutually interacting disturbance quantities and of the mean flow, with which the disturbance quantities also interact.

An "integral formulation" of the low-speed finiteamplitude instability problem for parallel flow and temporal disturbances, in which no assumption about the smallness of the amplification rates is made, is discussed by Stuart. ${ }^{8}$ The extension of this technique to a simultaneously developing mean wake flow interacting with spatially growing disturbances has recently been made by Ko, Kubota, and Lees. ${ }^{9}$ The gross features of the problem, such as the centerline velocity defect and the wake width in the finite-amplitude disturbance region, are found to be in good agreement with Sato and Kuriki's experiments ${ }^{10}$ on the unstable laminar wake behind a flat plate placed parallel to a low-speed uniform stream, even though the theory included only the interaction of the fundamental disturbance with the mean flow.

As a sequel to the previous work on a weakly nonlinear theory, ${ }^{4}$ this paper discusses a "strongly nonlinear theory" in terms of a von Kármán integral formulation with some discussion of the compressibility transformation. The same Reynolds splitting procedure is used as in earlier works ${ }^{4,9}$; the disturbance quantities are considered as spatially growing and oscillating with a real frequency, and averages are taken with respect to time. The over-all momentum, kinetic, and thermal energy balances, while obscuring the details of the problem, bring out its main physical features clearly. The disturbances and the mean flow interact in several ways. The more familiar mechanism, as in the lowspeed problem, is the role played by the Reynolds stresses in converting the mean-flow kinetic energy into the kinetic energy of the disturbances. While the viscous dissipation of the mean flow and of the disturbances contributes to a decrease in their respective kinetic energies, this dissipation also contributes to an increase in the thermal energy of the 
mean flow. The increase in disturbance kinetic energy through the action of the disturbance pressure gradients reflects a loss in thermal energy of the mean flow. The disturbance thermal energy equation does not play a role here since it averages out to zero. In the integral formulation, applied to the boundary-layer forms of the equations, the transport of disturbance thermal energy does not enter into the mean-flow thermal energy equation.

The integral form of the equations contains the mean-flow density in the convection terms; this explicitly renders the equations coupled. Implicit coupling appears through the time-averaged disturbance terms. As recently discussed by Laufer, ${ }^{11}$ two methods have been used to make an effective Howarth transformation of the compressible turbulent boundary-layer equations. One is the usual normal coordinate stretching, using the mean-flow density, but applied to an incomplete form of the equations, together with phenomenological assumptions about the turbulent transport terms. In the other, used by Laufer, ${ }^{11}$ the instantaneous density is used in the normal coordinate stretching which is applied to the original equations prior to any Reynolds splitting. Certain density-weighted variables are defined in this procedure and a Reynolds splitting is subsequently made for these variables. Laufer ${ }^{11}$ found it necessary to neglect the pressure fluctuations; in order to render his transformation effective, the temperature-velocity gradient correlations are also neglected.

In the present problem the mean-flow differential equations are in the same form as those for the compressible turbulent boundary layer without any phenomenological assumptions about the disturbances. The disturbance quantities, on the other hand, satisfy their respective conservation equations in which the linear instability form is the starting point. Phenomenological assumptions about the forms of the time-averaged disturbance quantities in the mean-flow equations are, therefore, unnecessary. It is known from the linear theory that the disturbance pressure fluctuations are just as important as fluctuations of other disturbance quantities, and that the mean of the product of disturbance temperature and velocity gradients is, in general, not zero. The compressibility transformations suggested by work on turbulent compressible shear flows are thus not applicable in their existing form. An attempt to obtain a satisfactory compressibility transformation for the present problem in its most general form appears futile. However, the usual Howarth transformation, using the mean-flow density, is found to be satisfactory in the restrictive consideration of integral relations of the von Kármán type discussed below.

\section{BASIC EQUATIONS}

Although the detailed distribution of the disturbances is not sensitive to the viscous terms in wake-type flows (thus leading to the consideration of "inviscid" disturbances ${ }^{4,12}$ ), retention of the disturbance viscous terms is essential in the energy balance in order to bring out the gross physical processes involved. These terms are retained in what follows, but the contributions from the fluctuations of the temperature-dependent viscosity and thermal conductivity are ignored. Of course, these latter contributions can always be included without diffculty; their presence is cumbersome, however, and contributes nothing to the essence of the problem.

The Prandtl boundary-layer approximations have been applied to the mean-flow equations given below. The continuity equation is

$$
\frac{\partial \bar{\rho}^{*} \bar{u}^{*}}{\partial x^{*}}+\frac{\partial\left(\bar{\rho}^{*} \bar{v}^{*}+\overline{\rho^{* \prime} v^{* \prime}}\right)}{\partial y^{*}}=0
$$

where $\bar{\rho}^{*}$ and $\rho^{* \prime}$ are the mean-flow and disturbance densities, respectively; $x^{*}$ is the longitudinal (streamwise) positional coordinate measured from the trailing edge of the body along the wake centerline, and $y^{*}$ is the normal positional coordinate measured from the wake centerline; $\bar{u}^{*}$ and $\bar{v}^{*}$ are the mean flow $x^{*}$ - and $y^{*}$-velocity components, respectively, and $v^{* \prime}$ is the disturbance $y^{*}$-component velocity. Here, an asterisk denotes a dimensional quantity and a bar over the disturbance quantities denotes the time average. By definition, the time average of a single disturbance quantity is zero.

The $x^{*}$ - and $y^{*}$-component mean-flow momentum equations are

$$
\begin{aligned}
\bar{\rho}^{*} & \left(\bar{u}^{*} \frac{\partial \bar{u}^{*}}{\partial x^{*}}+\bar{v}^{*} \frac{\partial \bar{u}^{*}}{\partial y^{*}}\right) \\
= & \frac{\partial}{\partial y^{*}}\left(\bar{\mu}_{1}^{*} \frac{\partial \bar{u}^{*}}{\partial y^{*}}-\left(\bar{\rho}^{*} \overline{u^{* \prime} v^{*^{\prime}}}+\overline{\rho^{*^{\prime}} u^{*^{\prime}} v^{*^{\prime}}}\right)\right) \\
& -\overline{\rho^{* \prime} v^{*^{\prime}}} \frac{\partial \bar{u}^{*}}{\partial y^{*}}
\end{aligned}
$$

and

$$
\frac{\partial \bar{p}^{*}}{\partial y^{*}}=-\frac{\partial}{\partial y^{*}}\left(\bar{\rho}^{*} \overline{v^{*^{\prime}}}+\overline{\rho^{* \prime} v^{*^{\prime 2}}}\right)
$$

respectively, where $\bar{\mu}_{1}^{*}$ is the viscosity coefficient, $u^{* \prime}$ is the disturbance $x^{*}$-component velocity, and $\bar{p}^{*}$ is the mean-flow pressure. A more convenient form of 
the $x^{*}$-component mean-flow momentum equation is

$$
\begin{aligned}
& \frac{\partial \bar{\rho}^{*} \bar{u}^{*^{2}}}{\partial x^{*}}+\frac{\partial \bar{\rho}^{*} \bar{v}^{*} \bar{u}^{*}}{\partial y^{*}}=\frac{\partial}{\partial y^{*}} \\
& \cdot\left(\bar{\mu}_{1}^{*} \frac{\partial \bar{u}^{*}}{\partial y^{*}}-\left(\bar{\rho}^{*} u^{*^{\prime}} v^{*^{\prime}}+\overline{\rho^{* \prime} u^{*^{\prime}} v^{*^{\prime}}}+\overline{\rho^{* \prime} v^{* \prime}} u^{*}\right)\right) .
\end{aligned}
$$

In the above $x^{*}$-component momentum equations, the streamwise pressure gradient term, $-\partial \bar{p}^{*} / \partial x^{*}$, does not appear for two reasons: The Prandtl boundary-layer approximations have been applied to the average of the disturbance quantities and it was assumed that the external pressure, $\bar{p}_{e}^{*}$, is constant. The possibility of self-induced pressure due to the growth of the wake requires separate consideration.

The mean-flow kinetic energy equation is obtained by multiplying (2) by $\bar{u}^{*}$, with the use of (1):

$$
\begin{aligned}
& \frac{\partial \bar{\rho}^{*} \bar{u}^{*} \bar{u}^{*^{2}} / 2}{\partial x^{*}}+\frac{\partial \bar{\rho}^{*} \bar{v}^{*} \bar{u}^{*^{2}} / 2}{\partial y^{*}} \\
& =\frac{\partial}{\partial y^{*}}\left(\bar{\mu}_{1}^{*} \frac{\partial \bar{u}^{*^{2}} / 2}{\partial y^{*}}-\overline{\rho^{* \prime} v^{*^{\prime}}} \frac{\bar{u}^{*^{2}}}{2}\right. \\
& \left.-\bar{u}^{*}\left(\bar{\rho}^{*} \overline{u^{*^{\prime}} v^{*^{\prime}}}+\overline{\rho^{*^{\prime}} u^{*^{\prime}} v^{*^{\prime}}}\right)\right)-\left(-\bar{\rho}^{*} \overline{u^{* \prime} v^{*^{\prime}}}\right. \\
& \left.-\overline{\rho^{* \prime}} \overline{u^{*^{\prime}} v^{*^{\prime}}}\right) \frac{\partial \bar{u}^{*}}{\partial y^{*}}-\bar{\mu}_{1}^{*}\left(\frac{\partial \bar{u}^{*}}{\partial y^{*}}\right)^{2} .
\end{aligned}
$$

The mean-flow thermal energy equation is written in terms of the static enthalpy which is related to the mean-flow internal energy via (8) below, augmented by the disturbance viscous dissipation terms:

$$
\begin{aligned}
& \frac{\partial \bar{\rho}^{*} \bar{u}^{*} C_{p} \bar{T}^{*}}{\partial x^{*}}+\frac{\partial \bar{\rho}^{*} \bar{v}^{*} C_{p} \bar{T}^{*}}{\partial y^{*}} \\
& =\frac{\partial}{\partial y^{*}}\left(\bar{k}^{*} \frac{\partial \bar{T}^{*}}{\partial y^{*}}-C_{p}\left(\overline{\rho^{*} v^{* \prime} T^{*^{\prime}}}+\overline{\rho^{* \prime} v^{* \prime} T^{*^{\prime}}}\right.\right. \\
& \left.\left.\quad+\overline{\rho^{*^{\prime}} v^{*^{\prime}}} \bar{T}^{*}\right)\right)+\bar{\mu}_{1}^{*}\left(\frac{\partial \bar{u}^{*}}{\partial y^{*}}\right)^{2} .
\end{aligned}
$$

$$
\begin{aligned}
& +\overline{\left(u^{* \prime} \frac{\partial p^{*^{\prime}}}{\partial x^{*}}\right.}+\overline{\left.v^{* \prime} \frac{\partial p^{* \prime}}{\partial y^{*}}\right)}+\bar{\mu}_{1}^{*}\left\{\frac { 4 } { 3 } \left[\overline{\left(\frac{\partial u^{* \prime}}{\partial x^{*}}\right)^{2}}\right.\right. \\
& \left.+\left(\overline{\left.\frac{\partial v^{* \prime}}{\partial y^{*}}\right)^{2}}-\overline{\left(\frac{\partial u^{* \prime}}{\partial x^{*}}\right)\left(\frac{\partial v^{* \prime}}{\partial y^{*}}\right)}\right]+\overline{\left(\frac{\partial u^{* \prime}}{\partial y^{*}}+\frac{\partial v^{* \prime}}{\partial x^{*}}\right)^{2}}\right\}
\end{aligned}
$$

where $C_{p}$ is the specific heat at constant pressure, $\bar{T}^{*}$ is the mean flow temperature, $\bar{k}^{*}$ is the thermal conductivity, and $T^{* \prime}$ is the disturbance temperature. The second viscosity coefficient, $\bar{\mu}_{2}^{*}$, which is supposed to account for the internal degree of freedom of molecular gases, ${ }^{13}$ is here omitted in the disturbance viscous dissipation terms. Again, the effect of the mean-flow pressure gradients does not appear in (6) because of the Prandtl boundary-layer approximations.

The thermal and caloric equations of state for the mean flow are

$$
\begin{gathered}
\bar{p}^{*}=R\left(\bar{\rho}^{*} \bar{T}^{*}+\overline{\rho^{* \prime}} \overline{T^{* \prime}}\right), \\
C_{p} \bar{T}^{*}=C_{v} \bar{T}^{*}+\frac{\overline{p^{*}}}{\bar{\rho}^{*}}-\frac{R \rho^{*^{\prime}} T^{*^{\prime}}}{\bar{\rho}^{*}},
\end{gathered}
$$

respectively, where $R$ is the gas constant and $C_{\mathrm{v}}$ is the specific heat at constant volume. The gas is assumed to be both thermally and calorically perfect.

Only the nonvanishing time-averaged disturbance kinetic energy will be needed in what follows for the energy balance. The time-averaged disturbance continuity, momentum, thermal energy, and state equations all vanish. The original, nonlinear equations for $u^{* \prime}$ and $v^{* \prime}$ are complicated and will not be given. The forms given in Ref. 4 are now augmented by the viscous terms, as previously discussed. Multiplying the $x^{*}$-disturbance momentum equation by $u^{* \prime}$ and the $y^{*}$-disturbance momentum equation by $v^{* \prime}$, with the use of the disturbance and mean flow continuity equations, we obtain the timeaveraged disturbance kinetic energy equation:

$$
\begin{aligned}
& \frac{1}{2} \frac{\partial}{\partial x^{*}}\left[\bar{\rho}^{*} \bar{u}^{*}\left(\overline{u^{*^{\prime 2}}+v^{*^{\prime 2}}}\right)\right]+\frac{1}{2} \frac{\partial}{\partial y^{*}}\left[\bar{\rho}^{*} \bar{v}^{*}\left(\overline{u^{* / 2}}+v^{*^{\prime 2}}\right)\right] \\
& =\frac{\partial}{\partial y^{*}}\left[\bar{\mu}_{1}^{*} \frac{\left.\partial \overline{\left(u^{*^{\prime 2}}+v^{*^{\prime 2}}\right.}\right) / 2}{\partial y^{*} u}+\frac{4}{3} \bar{\mu}_{1}^{*} \frac{\overline{\partial v^{*^{\prime 2}} / 2}}{\partial y^{*}}+\bar{\mu}_{1}^{*}\left(\overline{u^{* \prime} \frac{\partial v^{*^{\prime}}}{\partial x^{*}}}-\frac{2}{3} \overline{v^{* \prime}} \overline{\frac{\partial u^{* \prime}}{\partial x^{*}}}\right)\right. \\
& -\frac{1}{2}\left[\overline{\bar{\rho}^{*}} \overline{v^{*^{\prime}}\left(u^{*^{\prime 2}}+v^{*^{\prime 2}}\right)}+\bar{v}^{*} \overline{\rho^{*^{\prime}}\left(u^{*^{\prime 2}}+v^{*^{\prime 2}}\right)}+\overline{\left.\rho^{*^{\prime}} v^{*^{\prime}}\left(u^{*^{\prime 2}}+v^{*^{\prime 2}}\right)\right]}\right]-\left(\overline{u^{* \prime} \frac{\partial p^{*^{\prime}}}{\partial x^{*}}}+\overline{v^{* \prime}} \overline{\frac{\partial p^{*^{\prime}}}{\partial y^{*}}}\right) \\
& +\left(-\bar{\rho}^{*} \overline{u^{* \prime} v^{*^{\prime}}}-\overline{\left.\rho^{*^{\prime}} u^{*^{\prime}} v^{*^{\prime}}\right)} \frac{\partial \bar{u}^{*}}{\partial y^{*}}+\bar{\mu}_{1}^{*}\left\{\frac{4}{3}\left[\overline{\left(\frac{\partial u^{* \prime}}{\partial x^{*}}\right)^{2}}+\overline{\left(\frac{\partial v^{* \prime}}{\partial y^{*}}\right)^{2}}-\overline{\left(\frac{\partial u^{* \prime}}{\partial x^{*}}\right)\left(\frac{\partial v^{* \prime}}{\partial y^{*}}\right)}\right]+\overline{\left.\left(\frac{\partial u^{* \prime}}{\partial y^{*}}+\frac{\partial v^{* \prime}}{\partial x^{*}}\right)^{2}\right\} .}\right.\right.
\end{aligned}
$$

In (9) both the streamwise "diffusion"14 and "conduction" of disturbance kinetic energy are absent because of the boundary-layer approximations. Only the dominant mechanism of energy transfer 
from the mean flow, that through the rate of strain $\partial \bar{u}^{*} / \partial y^{*}$, is retained.

\section{VON KÁRMÁN INTEGRAL RELATIONS}

Let us introduce the following dimensionless variables:

$$
\begin{gathered}
x=\frac{x^{*}}{d}, \quad y=\frac{y^{*}}{d}, \quad u=\frac{\bar{u}^{*}}{\bar{u}_{e}^{*}}, \quad v=\frac{\bar{v}^{*}}{\bar{u}_{e}^{*}}, \quad \rho=\frac{\bar{\rho}^{*}}{\bar{\rho}_{e}^{*}}, \\
p=\frac{\bar{p}^{*}}{\bar{p}_{e}^{*}}, \quad T=\frac{\bar{T}^{*}}{\bar{T}_{e}^{*}}, \quad \mu_{1}=\frac{\bar{\mu}_{1}^{*}}{\bar{\mu}_{e 1}^{*}}, \\
u^{\prime}=\frac{u^{* \prime}}{\bar{u}_{e}^{*}}, \quad v^{\prime}=\frac{v^{* \prime}}{\bar{u}_{e}^{*}}, \quad \rho^{\prime}=\frac{\rho^{* \prime}}{\bar{\rho}_{e}^{*}}, \quad p^{\prime}=\frac{p^{* \prime}}{\bar{p}_{e}^{*}}, \\
T^{\prime}=\frac{T^{* \prime}}{\bar{T}_{e}^{*}},
\end{gathered}
$$

where the subscript $e$ refers to conditions at the edge of the wake and $d$ is a characteristic scale of body length.

The von Kármán integral form of the equations are obtained in terms of (10) after integrations across the width of the wake are carried out. Thus, (4) becomes

$$
\frac{d}{d x} \int_{-\infty}^{\infty} u(u-1) \rho d y=0 .
$$

The mean flow $y$-momentum equation, obtained from (3), becomes

$$
p=1-\left(\rho \overline{v^{\prime 2}}+\overline{\rho^{\prime} v^{\prime 2}}\right) .
$$

The kinetic energy integral equation of the mean flow, obtained from (5), becomes

$$
\frac{1}{2} \frac{d}{d x} \int_{-\infty}^{\infty} u\left(u^{2}-1\right) \rho d y=-I_{\Phi}-I_{r s}
$$

where the following integrals are now defined:

$$
I_{\Phi}=\frac{1}{\operatorname{Re}_{d}} \int_{-\infty}^{\infty} \mu_{1}\left(\frac{\partial u}{\partial y}\right)^{2} d y
$$

is the mean-flow dissipation integral (where $\operatorname{Re}_{d}=$ $\bar{\rho}_{e}^{*} \bar{u}_{e}^{*} d / \bar{\mu}_{e}^{*}$ is the Reynolds number based on the conditions at the outer edge of the wake and on the body length scale), and

$$
I_{r s}=\int_{-\infty}^{\infty}\left(-\rho \overline{u^{\prime} v^{\prime}}-\overline{\rho^{\prime} u^{\prime} v^{\prime}}\right) \frac{\partial u}{\partial y}
$$

is an integral representing the transfer of energy, due to the action of the Reynolds stresses, from the mean flow to the disturbances. Of course, both energy since $I_{\phi^{\prime}}>0$ always, and a corresponding increase in the mean-flow thermal energy takes place. $-I_{\Phi}$ and $-I_{r s}$ in (13) lead to a decrease in the mean-flow kinetic energy. The mean-flow thermal energy integral equation, obtained from (6), is

$\frac{d}{d x} \int_{-\infty}^{\infty} u(T-1) \rho d y=(\gamma-1) M_{e}^{2}\left(I_{\Phi}-I_{p^{\prime}}+I_{\phi^{\prime}}\right)$,

where $\gamma=C_{p} / C$, is the ratio of specific heats and $M_{e}=\bar{u}_{e}^{*} /\left(\gamma R \bar{T}_{e}^{*}\right)^{1 / 2}$ is the Mach number at the outer edge of the wake. The last two integrals on the right-hand side of (16) are

$$
I_{p^{\prime}}=\frac{1}{\gamma M_{e}^{2}} \int_{-\infty}^{\infty}\left(-\overline{u^{\prime} \frac{\partial p^{\prime}}{\partial x}}-\overline{v^{\prime} \frac{\partial p^{\prime}}{\partial y}}\right) d y
$$

which represents the action of the disturbance pressure gradients, and

$$
\begin{aligned}
& I_{\phi^{\prime}}= \frac{1}{\operatorname{Re}_{d}} \int_{-\infty}^{\infty} \mu_{1}\left\{\frac { 4 } { 3 } \left[\overline{\left(\frac{\partial u^{\prime}}{\partial x}\right)^{2}}+\overline{\left(\frac{\partial v^{\prime}}{\partial y}\right)^{2}}\right.\right. \\
&\left.-\left(\frac{\partial u^{\prime}}{\partial x}\right)\left(\frac{\partial v^{\prime}}{\partial y}\right)\right]+\left(\overline{\left.\frac{\partial u^{\prime}}{\partial y}+\frac{\partial v^{\prime}}{\partial x}\right)^{2}}\right\} d y
\end{aligned}
$$

which is the disturbance dissipation integral. The mean flow integral equations are augmented by the equations of state, obtained from (7)

$$
p=\rho T+\overline{\rho^{\prime}} \bar{T}^{\prime} \text {. }
$$

The dimensionless form of the caloric equation of state, (8), appears in the form

$$
T=\frac{T}{\gamma}+\left(\frac{p}{\rho}-\frac{\overline{\rho^{\prime} T^{\prime}}}{\rho}\right) \frac{(\gamma-1)}{\gamma}
$$

which is identical to (19).

The time-averaged disturbance kinetic-energy integral equation obtained from (9) becomes

$$
\begin{aligned}
\frac{1}{2} \frac{d}{d x} \int_{-\infty}^{\infty}\left[\rho u\left(\overline{u^{\prime 2}+v^{\prime 2}}\right)\right] & d y \\
& =I_{p^{\prime}}-I_{\phi^{\prime}}+I_{r s},
\end{aligned}
$$

where the integrals $I_{p^{\prime}}, I_{\phi^{\prime}}$, and $I_{\mathrm{ra}}$ are defined in (17), (18), and (15), respectively. The left-hand side of (20) represents the streamwise rate of change of the integral of the time-averaged, convected disturbance kinetic energy. If the action of the disturbance pressure gradients contribute to an increase in the disturbance kinetic energy, i.e., if $I_{p^{\prime}}>0$, then a corresponding decrease in the mean flow thermal energy takes place in (16). The disturbance viscous dissipation decreases the disturbance kinetic energy since $I_{\phi^{\prime}}>0$ always, and a corresponding increase in the mean-flow thermal energy takes place. These two mechanisms represent the interaction of the disturbance kinetic energy with the mean-flow thermal energy in a compressible fluid. The transfer of mean-flow kinetic energy to disturbance kinetic energy through the work done by the Reynolds 
stresses, $I_{r s}$, is the more familiar mechanism in an incompressible fluid.

The implicit interactions between the disturbance and the mean flow occur, for instance, through the presence of the mean-flow density in the convection terms and the dependence of the mean-flow pressure on the disturbances in the equation of state (19). However, these only affect the details of the problem. The main physical mechanisms of the coupling interactions are represented by $I_{r s}, I_{p^{\prime}}, I_{\phi^{\prime}}$, and $I_{\Phi}$.

The sum of the streamwise rate of change of the mean-flow kinetic and thermal energies effects a cancellation of the mean-flow viscous dissipation term $I_{\Phi}$. In the absence of mean-flow interaction with the disturbance, it is well known that such a sum produces an integral which is conserved. In the present problem, such a sum produces the source term $I_{\phi^{\prime}}$ and the sink (or source) term $I_{p^{\prime}}$ for the rate of change of the integral of the total convected mean-flow energy. These two terms are precisely the sink and source (or sink) terms, respectively, for the rate of change of the integral of convected, timeaveraged disturbance kinetic energy. When the mean flow and the disturbances are viewed as a single system, the "internal" sources and sinks cancel and the conserved integral

$$
\begin{aligned}
\int_{-\infty}^{\infty} & \left\{\rho u\left[\frac{T-1}{(\gamma-1) M_{e}^{2}}+\frac{1}{2}\left(u^{2}-1\right)\right]\right. \\
& \left.+\frac{1}{2}\left[\rho u\left(\overline{u^{\prime 2}+v^{\prime 2}}\right)\right]\right\} d y=\text { const }
\end{aligned}
$$

is produced.

\section{COMPRESSIBILITY TRANSFORMATION AND SHAPE ASSUMPTIONS}

The left-hand sides of the mean flow equations, (11), (13), and (16), appear particularly suitable for the standard Howarth transformation. In this case, the mean-flow density used is one in which the disturbance contributions to the mean flow pressure is included. Hence, the left-hand sides of (11), (13), and (16) will undergo a "fine" Howarth transformation. To effect a Howarth transformation upon the disturbance quantities, we now specify that the disturbance quantities under the integrals are to be evaluated locally. This is a feasible approximation, ${ }^{4,9}$ since for wake flows the mean-flow development time is expected to be much longer than the disturbancedisturbance and disturbance-mean-flow interaction times. Following Ko, Kubota, and Lees, ${ }^{9}$ the disturbances are assumed to consist of an unknown amplitude multiplying a shape function which is generated by the local linear theory. Here, the shape functions are those of the Lees and Gold spatial eigenfunctions. ${ }^{12}$ The disturbance effects are considered to order of the amplitude squared, $|A|^{2}$. In this context, to be consistent, the Howarth transformation applied to the disturbance quantities is also to the order $|A|^{2}$. The disturbance quantities thus undergo a "coarse" Howarth transformation. Such a transformation is also applied to the mean flow dissipation term, where $\rho \mu_{1}$ is not constant for $\mu_{1} \sim T$ because of the disturbance-generated meanflow pressure variations.

The shape assumption for the mean flow, after the "fine" Howarth transformation is applied, is then identical to the incompressible form. ${ }^{9}$ Let

$$
\eta=\frac{1}{b} \int_{y} \rho d y
$$

where $b(x)$ is now the Howarth-transformed local (e-folding) wake width. The mean-flow velocity is assumed to be of the form

$$
u(x, \eta)=1-V(x) \exp \left(-\eta^{2}\right),
$$

where $V(x)=1-u(x, 0)$ is the dimensionless meanflow velocity defect at the wake centerline. The mean-flow temperature is assumed to be of the form

$$
T(x, \eta)=1+T_{c}(x) \exp \left(-\eta^{2}\right),
$$

where $T_{c}(x)=T(x, 0)-1$ is the mean-flow temperature defect at the wake centerline.

The disturbance quantities are assumed to be of the form

$$
\begin{aligned}
\frac{u^{\prime}}{V}(x, \eta) & =A F_{1} \exp (-i \beta t)+\tilde{A} \tilde{F}_{1} \exp (i \beta t), \\
\frac{v^{\prime}}{V}(x, \eta) & =A \alpha \phi_{1} \exp (-i \beta t)+\tilde{A} \bar{\alpha} \tilde{\phi}_{1} \exp (i \beta t) \\
p^{\prime}(x, \eta) & =A \pi_{1} \exp (-i \beta t)+\tilde{A} \tilde{\pi}_{1} \exp (i \beta t) \\
\rho^{\prime}(x, \eta) & =A R_{1} \exp (-i \beta t)+\tilde{A} \tilde{R}_{1} \exp (i \beta t), \\
T^{\prime}(x, \eta) & =A \theta_{1} \exp (-i \beta t)+\tilde{A} \tilde{\theta}_{1} \exp (i \beta t)
\end{aligned}
$$

which makes the interaction between the disturbance and the mean flow to be of order $|A|^{2}$, with the harmonic terms excluded. The tilde indicates the complex conjugate. Accordingly, the relation

$$
\frac{d A}{d x}=i \alpha A+O|A|^{2}
$$

is used to evaluate the $x$ derivatives of the disturbance quantities locally. The eigenfunctions $F_{1}, \phi_{1}$, $\pi_{1}, R_{1}, \theta_{1}$ are obtained from the eigenvalue problem of the linear theory. Here, $\beta=\left(\beta^{*} d / \bar{u}_{e}^{*}\right)(b / d)$ is the (real) dimensionless frequency, $\beta^{*}$ is the physical frequency, the dimensionless time is $t=\left(t^{*} \bar{u}_{0}^{*} / d\right)$ $\cdot(d / b), \alpha=\left(\alpha^{*} d\right)(b / d)$ is the dimensionless wave- 
number (complex for the spatial-instability problem), and $\alpha^{*}$ is the dimensional wavenumber.

The coarse Howarth-transformed eigenvalue problem, in terms of $\pi_{1}$, is

$\pi_{1}^{\prime \prime}-2 \frac{w}{w-c} \pi^{\prime}-\alpha^{2} T^{2}\left(1-\frac{M^{2}(w-c)^{2}}{T}\right) \pi_{1}=0$

with the boundary conditions

$$
\begin{gathered}
\pi_{1}(0)=0, \\
\pi_{1}^{\prime}+\alpha T \Omega^{1 / 2} \pi_{1} \rightarrow 0 \text { as } \eta \rightarrow \infty,
\end{gathered}
$$

where $\Omega=1-M^{2}(w-c)^{2} / T, w=(u-1) / V$, $c=(\beta / \alpha-1) / V$, and $M^{2}=V^{*^{2}} / \gamma R \bar{T}_{e}^{*}$. The first of the boundary conditions (28) is that which gives the most unstable disturbance, corresponding to the "antisymmetrical" disturbances, ${ }^{12}$ and is used in preference to $\pi_{1}^{\prime}(0)=0$, which corresponds to "symmetrical" disturbances.

By using (25), the equation of state (19) becomes

$$
p=\rho T+|A|^{2}\left(R_{1} \tilde{\theta}_{1}+\tilde{R}_{1} \theta_{1}\right)
$$

and the $y$-momentum equation (12) becomes

$$
p=1-\gamma M_{e}^{2} V^{2} \rho|A|^{2}\left|\alpha \phi_{1}\right|^{2},
$$

both to order $|A|^{2}$. Then the $\rho \mu_{1}$ product, with a linear temperature-viscosity relation assumed, becomes

$$
\begin{array}{r}
\rho \mu_{1}=C_{\rho} T=C\left\{1-|A|^{2}\left[\left(R_{1} \tilde{\theta}_{1}+\tilde{R}_{1} \theta_{1}\right)\right.\right. \\
\left.\left.+2 \gamma M_{e}^{2} V^{2}\left|\alpha \phi_{1}\right|^{2} / T\right]\right\},
\end{array}
$$

where $C$ is the Chapman-Rubesin constant. This will be used in the mean-flow dissipation integral.

Since here we deal only with disturbance effects to order $|A|^{2}$, only the fundamental component of the disturbances are included. The time-averaged triple disturbance quantities are, therefore, zero (since the second harmonic component would have to interact with the fundamental to produce such quantities). Also, the time-averaged quadruple disturbance quantities would occur as part of the streamwise diffusion effect were it not for the boundary-layer approximations, do not enter in the order $|A|^{2}$ consideration.

With the use of (22)-(25), the mean-flow $x$-momentum equation (11) becomes

$$
V b\left(C_{2} V-C_{1}\right)=C_{D} / 2,
$$

where $C_{1}=\pi^{1 / 2}, C_{2}=(\pi / 2)^{1 / 2}$, and $C_{D}$ is the drag coefficient of the body. Equation (32) is identical to the incompressible form ${ }^{9}$ when the boundarylayer approximations are also applied to the timeaveraged disturbance quantities. The mean-flow kinetic energy equation (13) becomes

$$
\begin{aligned}
& \frac{1}{2} \frac{d}{d x}\left[b V\left(3 C_{2} V-2 C_{1}-C_{3} V^{2}\right)\right] \\
& \quad=-\frac{C}{\operatorname{Re}_{d}} \frac{4 V^{2}}{b} I_{\Phi}-V^{3}|A|^{2} I_{r s},
\end{aligned}
$$

where $C_{3}=(\pi / 3)^{1 / 2}$ and

$$
\begin{gathered}
I_{\Phi}=I_{\Phi 1}-|A|^{2}\left(I_{\Phi 2}+2 \gamma M_{c}^{2} V^{2}|\alpha|^{2} I_{\Phi 3}\right), \\
I_{\Phi 1}=C_{4}=(\pi / 8)^{1 / 2} \\
I_{\Phi 2}=\int_{-\infty}^{\infty}\left(R_{1} \tilde{\theta}_{1}+\tilde{R}_{1} \theta_{1}\right) \eta^{2} \exp \left(-2 \eta^{2}\right) d \eta, \\
I_{\Phi 3}=\int_{-\infty}^{\infty}\left|\phi_{1}\right|^{2}\left[1+T_{c} \exp \left(-\eta^{2}\right)\right]^{-1} \eta^{2} \\
\cdot \exp \left(-2 \eta^{2}\right) d \eta, \\
I_{r s}=\int_{-\infty}^{\infty}\left(F_{1} \tilde{\alpha} \tilde{\phi}_{1}+\tilde{F}_{1} \alpha \phi_{1}\right)\left[1+T_{c}\right. \\
\left.\cdot \exp \left(-\eta^{2}\right)\right]^{-1} 2 \eta \exp \left(-\eta^{2}\right) d \eta .
\end{gathered}
$$

The integrals $I_{\Phi_{2}}, I_{\Phi_{3}}$, and $I_{r s}$ are functions of $\left(V, T_{c}\right)$ for a given physical frequency $\beta^{*}$. The lefthand side of (33) is again identical to the low-speed form. The mean-flow thermal energy equation becomes

$$
\begin{aligned}
& \frac{d}{d x}\left[b T_{c}\left(C_{1}-C_{2} V\right)\right]=(\gamma-1) M_{c}^{2} \\
& \quad \cdot\left(\frac{C}{\operatorname{Re}_{d}} \frac{4 V^{2}}{b} I_{\Phi}+\frac{C}{\operatorname{Re}_{d}} \frac{2 V^{2}|A|^{2}}{b} I_{\phi^{\prime}}-\frac{V|A|^{2}}{\gamma M_{c}^{2}} I_{v^{\prime}}\right),
\end{aligned}
$$

where

$$
\begin{gathered}
I_{\phi^{\prime}}=\int_{-\infty}^{\infty}\left\{\left[1+T_{c} \exp \left(-\eta^{2}\right)\right]^{2}\left(\frac{4}{3}\left|F_{1}\right|^{2}+\left|\alpha \phi_{1}\right|^{2}\right)|\alpha|^{2}\right. \\
+\left[1+T_{c} \exp \left(-\eta^{2}\right)\right]\left[\alpha^{2} \phi_{1} \widetilde{F}_{1}^{\prime}-\tilde{\alpha}^{2} \tilde{\phi}_{1} F_{1}^{\prime}\right. \\
\left.+i \frac{2}{3}|\alpha|^{2}\left(\widetilde{F}_{1} \phi_{1}^{\prime}-F_{1} \tilde{\phi}_{1}^{\prime}\right)+\left(\frac{4}{3}\left|\alpha \phi_{1}^{\prime}\right|^{2}+\left|F_{1}^{\prime}\right|^{2}\right)\right\} d \eta, \quad(36) \\
I_{p^{\prime}}=-\int_{-\infty}^{\infty}\left\{\left[1+T_{c} \exp \left(-\eta^{2}\right)\right]\left(\alpha \widetilde{F}_{1} \pi_{1}-\tilde{\alpha} F_{1} \tilde{\pi}_{1}\right) i\right. \\
\left.+\alpha \phi_{1} \tilde{\pi}_{1}^{\prime}+\tilde{\alpha} \tilde{\phi}_{1} \pi_{1}^{\prime}\right\} d \eta .
\end{gathered}
$$

$I_{\phi^{\prime}}$ and $I_{p^{\prime}}$ are again functions of $\left(V, T_{c}\right)$ for a fixed $\beta^{*}$. Equation (35), of course, has no counterpart in the low-speed theory. Finally, the disturbance kinetic equation (20) becomes

$$
\begin{aligned}
& \frac{d}{d x}\left[|A|^{2} V^{2} b\left(I_{k e 1}-V I_{k e 2}\right)\right] \\
& =V|A|^{2}\left(V^{2} I_{r s}+\frac{1}{\gamma M_{e}^{2}} I_{p^{\prime}}-\frac{C}{\operatorname{Re}_{d}} \frac{2 V}{b} I_{\phi^{\prime}}\right),
\end{aligned}
$$

where

$$
\begin{gathered}
I_{k e 1}=\int_{-\infty}^{\infty}\left(\left|F_{1}\right|^{2}+\left|\alpha \phi_{1}\right|^{2}\right) d \eta=1, \\
I_{k \theta 2}=\int_{-\infty}^{\infty}\left(\left|F_{1}\right|^{2}+\left|\alpha \phi_{1}\right|^{2}\right) \exp \left(-\eta^{2}\right) d \eta .
\end{gathered}
$$


Following Ref. 9, if $|A|^{2}$ is to represent the (Howarth) integral of the averaged disturbance kinetic energy (normalized by $V$ ), then $I_{k e 1}$ is set equal to unity.

The conserved integral (21) now becomes

$b T_{c}\left(C_{1}-C_{2} V\right)+(\gamma-1) M_{s}^{2} b V\left[\frac{1}{2}\left(-2 C_{1}+3 V C_{2}\right.\right.$

$\left.\left.-V^{2} C_{3}\right)+|A|^{2} V\left(I_{k e 1}-V I_{k e 2}\right)\right]=$ const.

The system of equations (32), (33), (35), and (37) includes three simultaneous first-order ordinary differential equations and one algebraic equation for the four unknowns $V, T_{c}, b$, and $|A|^{2}$. However, (39) can be used in place of the mean-flow thermal energy equation (37), the system then consists of two simultaneous first-order ordinary differential equations with two supplementary algebraic equations. After specifying the initial values of the four unknowns, the system then describes the nonlinear interaction problem.

\section{CONCLUDING REMARKS}

It has been our aim to show how the finiteamplitude disturbances and their interaction with the mean flow in certain experimentally observed unstable high-speed wake flows can be described in the simplest way without making the assumption that the disturbance amplification rates are small. A von Kármán integral formulation is obtained for the two-dimensional compressible problem. Although it is expected a priori that the compressibility transformation will not yield the corresponding incompressible problem because of the compressible disturbances, such a transformation considerably simplifies the mean-flow description. It is shown that, in addition to the mean-flow wake width, centerline velocity defect, and the disturbance amplitude (which are governed by one algebraic equation and two first-order differential equations), compressibility introduces at least one additional parameter: the mean-flow centerline temperature defect. The additional equation, because of over-all energy conservation, is an algebraic relation; this emphasizes the role of exchanges between kinetic and thermal energies.

Such simplifying assumptions as constant external pressure, boundary-layer approximations on the averaged disturbance quantities, and the neglect of higher harmonic components in the disturbanceshape assumption, can always be removed; they are not necessary assumptions and do not alter the deductions already obtained. For purposes of the inclusion of the higher harmonic components in the disturbance-shape assumptions the recent compressible weakly nonlinear theory ${ }^{4}$ can be used as a guide.

At this stage, future directions for the nonlinear or incipient-transition region appear to be twofold. One is the numerical analysis, which is important, but is, however, a problem in itself. The application of this theory to specific experimentally observed problems is reported elsewhere. ${ }^{15}$ The other is conceptual and is in regard to the inclusion of disturbances other than those oscillating at the peak-signal frequency. The inclusion of a spectrum of disturbances, or the "finite bandwidth" problem, appears to be more important for the compressible wake in that the observed spectrum surrounding the peak frequency is much broader than the low-speed problem. 'The present formulation is sufficiently simple so that its framework can be used to explore the possibilities of incorporating a spectrum of disturbances. The spectral problem, also needs to be grappled with at the weakly nonlinear level. The analysis of events subsequent to the nonlinear region, however, still awaits guidance from detailed experimental information.

\section{ACKNOWLEDGMENTS}

The research reported in this paper is supported in part by the National Science Foundation through Grant GK-10008 at Brown University (J.T.C.L.) and in part by the United States Army Research Office and the Advanced Research Projects Agency through Contract No. DA-31-124-ARO(D)-33 at the California Institute of Technology (L.L.).

\footnotetext{
${ }^{1}$ W. Behrens, AIAA J. 5, 2135 (1967).

2 W. Behrens, AIAA J. 6, 225 (1968).

${ }^{3}$ R. G. Batt and T. Kubota, AIAA J. 7, 2064 (1969).

4 J. T. C. Liu, Phys. Fluids 12, 1763 (1969).

${ }^{5}$ J. T. Stuart, J. Fluid Mech. 9, 353 (1960).

${ }^{6}$ J. Watson, J. Fluid Mech. 9, 371 (1960).

7 J. Watson, J. Fluid Mech. 14, 211 (1962).

${ }^{8}$ J. T. Stuart, J. Fluid Mech. 4, 1 (1958).

$\checkmark$ D. R. S. Ko, T. Kubota, and L. Lees, J. Fluid Mech. 40, 315 (1970).

10 H. Sato and K. Kuriki, J. Fluid Mech. 11, 321 (1961).

11 J. Laufer, AIAA J. 7, 706 (1969).

${ }_{12} \mathrm{~L}$. Lees and H. Gold, in Fundamental Phenomena in Hypersonic Flow, edited by J. G. Hall (Cornell University Press, Ithaca, New York, 1966), p. 310.

${ }^{13}$ See, for instance, H. S. Tsien, in Fundamentals of Gas Dynamics, edited by H. W. Emmons (Princeton University Press, Princeton, New Jersey, 1958), p. 14 for further discussion of accounting for this mechanism through the introduction of vibrational energy lag.

${ }_{14}$ This effect is also absent when only the fundamental disturbance component is used in the subsequent shape assumptions.

${ }_{15}$ P. M. Gururaj and J. T. C. Liu, Division of Engineering Report, Brown University, NSF GK-10008/1 (1970).
} 